\title{
Partizipation migrantisch markierter Bürgerinnen in der Süddeutschen Zeitung - eine diskursanalytische Sondierung
}

\author{
Madlen Pilz \\ Leibniz Institute for Research on Society and Space (IRS), 15537 Erkner, Germany \\ Correspondence: Madlen Pilz (madlen.pilz@leibniz-irs.de)
}

Received: 14 May 2019 - Revised: 4 April 2020 - Accepted: 27 April 2020 - Published: 1 July 2020

\begin{abstract}
Kurzfassung. Based on the discourse analysis of articles collected between 2010 and 2016 from the Süddeutsche Zeitung - a leading local and German national newspaper - my aim is to reconstruct the central conditions limiting or enabling the participation of those citizens in the public discourse who are generally constructed as „migrants“. Therefore, I analyse the central elements of the discourse around the subject migration/integration. My analysis is guided by the ,postmigrant debate', in particular by the approaches of the ,differential inclusion " of migrantised groups and their ,struggles of migration" combining it with critical race debates. My aim is to outline the different discursive ways that allow migrantised citizens to participate in public meaning making, and the ways, they use to contest majoritarian views. My analysis reveals their critical reconsideration of the system of differentiated inclusion, which is organizing the majoritarian discourse and ,migrants' everyday lives. While the journalistic strategy exemplified within the analysis is working in support of the ,migrant's perspectives', it simultaneously acts to normalize majoritarian position contra migration.
\end{abstract}

\section{Einführung}

„Ich identifiziere mich mit der Stadt und fühle mich hier beheimatet", sagt Erciyas. Dass er Lösungen für Münchens Verkehrskonzepte suchen und Ampeln planen kann, aber nicht den Oberbürgermeister und Stadtrat wählen darf, das hält er für eine Schande der Demokratie (SZ, 23. Februar 2016).

Die zitierte Textpassage aus der Süddeutschen Zeitung wirft einige Fragen auf, mit denen ich mich in diesem Bei$\operatorname{trag}^{1}$ systematischer beschäftigen möchte: Warum wird Erciyas’ Aussage „Ich fühle mich hier beheimatet“ direkt und seine Kritik an der Demokratie indirekt zitiert? Was bedeu-

\footnotetext{
${ }^{1}$ Der vorliegende Artikel basiert auf Analysen, die im Rahmen des Forschungsprojekts „Lokal gestrandet, global vernetzt? Umgang mit Vielfalt an den gesellschaftlichen Rändern der postmigrantischen Stadt. Eine vergleichende Untersuchung in München und Leipzig“ am Leibniz-Institut für Länderkunde von August 2016Mai 2019 erfolgten.
}

tet die journalistisch gesetzte Verwendung von direktem und indirektem Zitat für seine Sprechposition ${ }^{2}$ im Zeitungsdiskurs? Was lässt sich daraus über die diskursiven Aushandlungen zum Thema Migration/Integration in der Süddeutschen Zeitung ableiten? Mein zentrales Anliegen in diesem Artikel ist es, die diskursiven Bedingungen und Formen der Partizipation migrantisch markierter Bürgerinnen ${ }^{3}$ am öffentlichen Diskurs am Beispiel ausgewählter Zeitungsartikel aus der Süddeutschen Zeitung aus den Jahren 2010 bis 2016 zu untersuchen. Ich möchte in diesem Beitrag rekonstruieren, welche diskursiven Bedingungen für die Partizipation migrantisch markierter Bürgerinnen an den öffentlichen Aushandlungen im Zeitungsdiskurs konstituierend sind. Welche Äußerungen finden Eingang und werden wie im Zeitungs-

\footnotetext{
${ }^{2}$ Im Artikel benutze ich zur Bezeichnung beider Geschlechter die weibliche Variante und nur im konkreten Sprechmoment eines männlichen Akteurs die männliche Form.

${ }^{3}$ Ich benutze den Begriff der Sprecherin und Bürgerin synonym und mit der Absicht, auf die Verwobenheit beider Kategorien, das Eingebettetsein des Sprechens im Zeitungsdiskurs in den Alltag, zu verweisen.
} 
diskurs journalistisch bewertet, kommentiert und gerahmt? Welche journalistischen Mittel werden genutzt, um spezifische Aussagen anzuerkennen, zu unterstützten oder abzulehnen und zu delegitimieren? Welche Sprechpositionen können migrantisch markierte Bürgerinnen legitimierweise im Diskurs einnehmen? Was lässt sich daraus über das Verhältnis zwischen Mehrheits- und Minderheitsgesellschaft aussagen? Mit diesen Fragen positioniere ich meinen Beitrag im Feld der postmigrantischen Perspektive, die danach fragt, wie eine durch Migration strukturierte Gesellschaft, die diese Tatsache für sich anerkennt, die zunehmende Pluralisierung und Heterogenisierung der Gesellschaft auf politischer, struktureller, diskursiver und subjektiver Ebene aushandelt (Canan und Foroutan, 2016; Foroutan, 2018). In meiner Analyse werde ich die Herangehensweise der postmigrantischen Perspektive mit dem Instrumentarium der Diskursforschung kombinieren. Von diesen Vorüberlegungen ausgehend möchte ich thesenhaft vorab formulieren, dass in den öffentlichen Aushandlungen neben partiellen Inklusionen nach wie vor die Stigmatisierung von Migration verhandelt wird, die Spielhaus $(2012,2018)$ als Hadern bzw. Obsession der Gesellschaft mit Migration bezeichnet.

Meine Analyse ist wie folgt aufgebaut: Im 2. Kapitel werde ich die postmigrantische Perspektive und die damit verbundenen Konzepte, die meine interpretative Folie bilden, darstellen. Im 3. Kapitel gehe ich näher auf mein empirisches Material und den spezifischen diskursanalytischen $\mathrm{Zu}$ griff ein. Im 4. Kapitel umreiße ich in knapper Form den diskursiven Kontext. Im 5. Kapitel stelle ich am Beispiel ausgewählter Textpassagen meine Analysen der zentralen Elemente des Diskurses, genauer gesagt, die Verknüpfung von Themen und Begriffen, von Äußerungsmodalitäten und Sprechpositionen vor, die die diskursiven Aushandlungen zum Gegenstand Migration/Integration in der Süddeutschen Zeitung charakterisieren.

\section{Theoretische Linien}

In der postmigrantischen Perspektive wird „Migration“ als ein Forschungsfeld konzipiert, das alle Fragen und Prozesse der Gesellschaft und ihres Wandels berührt. „Migration" wird somit zur zentralen Beobachtungskategorie und zum analytischen Ausgangspunkt gesellschaftlicher Prozesse (Broden und Mecheril, 2007; Bojadžijev und Römhild, 2014, 2017). Diesen Perspektivwechsel möchte ich mit Blick auf die diskursiven Aushandlungen im Zeitungsdiskurs nachvollziehen, wenn ich einerseits die Bedingungen der Partizipation migrantisch markierter Bürgerinnen am Zeitungsdiskurs untersuche, und andererseits analysiere, wie sie diese Zeitungsräume nutzen, d. h. welche Themen sie einbringen, welche Gegendiskurse sie entwickeln, welche Diskussionen und Dispute im Sinne von „Kämpfen der Migration“ sie austragen. Zur Interpretation meines empirischen Materials werden die in der postmigrantischen Debatte entwickelten Kon- zepte der „differentiellen Inklusion“ und der „Kämpfe der Migration" in Kombination mit Fragen der rassismuskritischen Analysen leitend sein.

Somit ist der Ausgangspunkt meiner Überlegungen der Prozess der Verlagerung und Vervielfältigung der Grenzregime in den „Innenraum der Nationen“, der den Alltag mit Blick auf Migration mit zahlreichen Grenzziehungen fragmentiert und ein System der differentiellen Inklusion produziert (Balibar, 2009; Mezzadra und Neilson, 2013). Daraus resultiert ein Verständnis von Integration als einem komplexen Wechselverhältnis von strategischen Ein- und Ausschlüssen, die - sich ergänzend - ineinander übergehen. Diese tragen dazu bei, Migration zu filtern, zu lenken und letztlich zu kontrollieren (ebd., 164 f.). Für migrantisch markierte Bürgerinnen ergeben sich daraus reale soziale Effekte, die Anerkennungsprozesse sowie die Teilhabeoptionen im sozialen, ökonomischen und politischen Feld strukturieren. Ziel meines Beitrags ist es somit, den Fokus auf die an diesen „Alltagsgrenzen“ stattfindenden „Kämpfe der Migration“ zu richten, die die „Initiierung einer Konfliktbeziehung zwischen Migration und den Versuchen ihrer Kontrolle" darstellen (Scheel, 2015). Da dieses System der Grenzziehungen weder gleichermaßen für jeden wirksam noch sichtbar ist, geht es hier um die meist unsichtbaren Kämpfe rund um die im Alltäglichen wirksam werdenden Grenzziehungen, die in den Büros mit Sekretärinnen, Amtsleiterinnen, Arbeitgeberinnen, Vermieterinnen und in privaten Räumen ausgetragen werden (Scheel, 2015; Riedner, 2018). Sie basieren auf den von de Certeau (1988) beschriebenen Praktiken der Aneignung und Rekodierung der mehrheitsgesellschaftlichen Kontrollinstrumente z. B. einem geschickten Ausnutzen der Situation, der Elemente im „Terrain“ des „Anderen“, um die eigenen Spielräume zu erweitern (ebd.). Diese Kämpfe stellen eine Praxis der Selbstermächtigung dar und hinterfragen den gesellschaftlichen Status quo und die mehrheitsgesellschaftliche Autorität (Scheel, 2015). Sie widmen sich Fragen der Teilhabe, des Zugangs zu Ressourcen und Rechten sowie der Anerkennung und der gesellschaftlichen Positionierung auf der Grundlage ethnisierender Zuschreibungen (Riedner, 2018).

Im Kern rückt dieser Fokus auf das System der ,differentiellen Inklusion“ und die „Kämpfe der Migration“ für meine Analyse das spezifische Wechselspiel von Inklusion und Grenzziehung auf Grundlage der migrantischen Markierung in den Blick. Er macht deutlich, dass die migrantische Markierung eine Praxis der Hierarchisierung von Gesellschaft ist, die in der strategischen „Veranderung“ und „Minorisierung“ spezifischer Gruppen durch Akte der Rassifizierung und Diskriminierung besteht, die den ,Anderen“ erst herstellen (Broden und Mecheril, 2010; Velho, 2010; Espahangizi et al., 2016). Er verweist darauf, dass durch die Debatten, die in der Öffentlichkeit unter dem Schlagwort Migration geführt werden und sich um Herkünfte, kulturelle Differenzen und Defizite drehen, die eigentliche Problematik in den Hintergrund rückt - die der eingeschränkten substantiellen und for- 
mellen Rechte für die Betroffenen im Alltag, die Bestandteil dieser rassistischen Akte der „Minorisierung“ sind.

Für meine vor dem Hintergrund postmigrantischer Fragestellungen durchgeführte Diskursanalyse der Aushandlungen von Migration/Integration in den Artikeln der Süddeutschen Zeitung bedeutet dies, zu untersuchen, wie das System der differentiellen Inklusion im Zeitungsdiskurs von Journalistinnen als Vertreterinnen des Mehrheitsdiskurses und migrantisch markierten Bürgerinnen reflektiert und verhandelt wird. Welche alltäglichen ,Kämpfe der Migration“ werden Teil der öffentlichen Aushandlung im Zeitungsdiskurs? Welches spezifische Verhältnis zwischen Mehrheitsgesellschaft und migrantisch Markierten lässt sich aus dem Zeitungsdiskurs rekonstruieren?

\section{Diskursanalytischer Zugang}

Für meine Analyse habe ich zehn Artikel aus einer Materialsammlung aus der Süddeutschen Zeitung aus den Jahren 2010 bis 2016 ausgewählt. ${ }^{4}$ Die Kriterien für die vorliegende Auswahl waren inhaltlicher wie auch struktureller Art: In der Auswahl sollten erstens die zentralen Themen der postmigrantischen Debatte verhandelt werden, also Fragen der Zugehörigkeiten und Differenz, der formellen, sozialen und ökonomischen Grenzziehungen und der Strategien der Migrantisierung. Zweitens sollte auch die Perspektive Migrantisierter repräsentiert sein. Die ungleichmäßige Verteilung der Artikel über die Jahre ist zum großen Teil auf journalistische Konjunkturen ${ }^{5}$ zurückzuführen, wodurch aus spezifischen Anlässen Artikelserien zu einem Themenfeld in kurzer Folge erscheinen. Eine Reihe von Artikeln zu spezifischen Diskursfeldern konnte nicht in die Analyse aufgenommen werden, da sie häufig nur eines der beiden Auswahlkriterien erfüllten oder hier eine sehr auf ein spezifisches Thema fokussierte Aushandlung geführt wurde, das von meinem eigentlichen Analysefokus in diesem Beitrag zu weit weggeführt hätte. ${ }^{6}$ Die Beschränkung auf zehn Artikel für den fol-

\footnotetext{
${ }^{4}$ Die Materialsammlung wurde im Rahmen des Projekts (Fußnote 1) angelegt und umfasst insgesamt 725 Artikel zu München. Der Artikelpool wurde aus dem digitalen Archiv der SZ mit Hilfe der Suchfunktion mit sieben unterschiedlichen Suchwörtern, teilweise auch in Kombination erstellt. Als das erfolgreichste Suchwort erwies sich „Migration“, das ca. 75\% des Materialpools erbrachte. Das lässt vermuten, dass Migration in der Süddeutschen Zeitung mehrheitlich als gesondertes (Problem-)Feld angesehen wird und nicht als Teil eines gemeinsamen Stadtalltags.

${ }^{5}$ Dazu gehört beispielsweise die 2011 in der SZ veröffentlichte Serie „Migranten in München“, die am selben Tag erschien wie die Besprechung der Studie des Sinus-Instituts und des Münchner Statistischen Amts zur Integration Münchner Migrantinnen (SZ, 14. März 2011). Aus dieser Serie wurden drei Artikel in die Auswahl aufgenommen, die das Spektrum in seiner Bandbreite abstecken.

${ }^{6}$ In der Auswahl nicht berücksichtigt wurden deshalb beispielsweise die Artikel zum Bahnhofsviertel (2010-2016), zu den bul-
}

genden Beitrag erfolgte aus Gründen der Überschaubarkeit, was nach diskursanalytischer Logik möglich ist, da Diskurse regelhaft auf der Grundlage des jeweils gültigen Wissens formiert sind (Foucault, 1994; Diaz-Bone, 2002; 2006; Jäger und Jäger, 2007) und die Regeln somit auch aus wenigen, einzelnen Sprechakten rekonstruiert werden können.

Meine diskursanalytische Vorgehensweise beruht auf einem zweistufigen Verfahren: Im ersten Schritt wurden die Artikel in Form einer Strukturanalyse nach Jäger (2015) in den Blick genommen. Darauf aufbauend erfolgte in einem zweiten Schritt am Beispiel ausgewählter Textpassagen die Analyse der Formationsregeln auf inhaltlicher und struktureller Ebene des Diskurses (Diaz-Bone, 2002, 2006; Bauriedl, 2007). In diesem Beitrag werde ich meine Analysen der vier zentralen Diskurselemente der Aushandlung von Migration/Integration unter Partizipation Migrantisierter beispielhaft darstellen. Die zentralen Inhalt und Struktur eines Diskurses organisierenden Elemente (Diaz-Bone, 2002, 2006; Bauriedl, 2007) sind (1) der Gegenstand des Diskurses, im vorliegenden Fall die Verhandlung von Migration/Integration, (2) die Begriffe, welche spezifische Perspektiven, Klassifizierungen und Positionen in diesem diskursiven Feld markieren, (3) die Äußerungsmodalitäten, d. h. die spezifischen Bedingungen, Eigenschaften, unterschiedlichen Formen der Subjektivierung und Akteurinnenschaft, die die Teilnahme am Diskurs charakterisieren und (4) die Themen, die das spezifische Feld der diskursiven Aushandlungen umfassen. Diese Elemente werden in der diskursiven Praxis hervorgebracht und ihre Rekonstruktion und Beschreibung ermöglicht Einblicke in die Regelhaftigkeiten, die einem Diskurs zu einer bestimmten Zeit zugrunde liegen.

\section{Diskursiver Kontext}

In der Diskursforschung wird der Zeitungsdiskurs als Teil der medialen Diskursebene neben die akademische, politische, wirtschaftliche oder alltägliche Diskursebene gestellt und als öffentlicher Interdiskurs bezeichnet. Seine Aufgabe ist es, zwischen der Öffentlichkeit und verschiedenen Spezialdiskursen zu vermitteln, weshalb er durch gegenseitige Anleihen und Zitierungen mit den übrigen Diskursebenen verflochten ist (Jäger, 2015). Der Zeitungsdiskurs in der Süddeutschen Zeitung ist der Öffentlichkeit (trotz interaktiver Kommentarfunktion im Internet) nur passiv zugänglich und wird von den Eliten des Zeitungsdiskurses kontrolliert und beschränkt (Lanz, 2007), d. h. eine Selektion sowie Kommentierung der Interviewpartnerinnen, Gewährspersonen, Themen und Aussagen findet durch Redakteurinnen und Journalistinnen statt. Charakteristisch für die Süddeutsche Zeitung ist, dass sie politisch im linksliberalen Spek-

garischen Bettlerinnen (2011-2012 und 2014) und Tagelöhnerinnen (2012-2016) und zum „Neubau eines Islamzentrums“ in München (2012-2016). 
trum verortet wird $^{7}$, zu den großen nationalen Qualitätszeitungen zählt, ein breites Lesepublikum adressiert und auch in der journalistischen Branche als Leitmedium fungiert (Blum, 2011). Somit besitzen die Diskursivierungen der Münchner Ereignisse auch auf der nationalen Skala einen meinungsbildenden Referenzcharakter.

Insgesamt zeichnet sich der Zeitungsdiskurs in den gesichteten Artikeln in der Süddeutschen Zeitung zwischen 2010 und 2016 zum Gegenstand Migration/Integration grob skizziert durch drei Diskursfelder aus: interkulturelle Vielfalt, Probleme mit ,migrantischen“ Mitbürgerinnen und erwünschte „Migrantinnen“ in München. In die erste Rubrik fallen Berichte über interkulturelle Stadtfeste, Migrantinnenvereine und Kulturzentren. Das zweite Diskursfeld ist breiter gefächert und beinhaltet z. B. den Themenkomplex Schule mit Fokus auf „die schwer erreichbaren“ Schülerinnen und Eltern, soziale Probleme oder patriarchale Ehebeziehungen. Die Probleme sind im Zeitungsdiskurs in einigen Münchner Neubauvierteln sowie im Bahnhofsviertel verortet. Letzteres ist außerdem speziell durch die Verschränkung mit den Diskursfeldern migrantische Ökonomie, Kriminalität, Armutsmigration, Sozialtourismus und „urbane Orientalisierung“ gekennzeichnet. Für das dritte Diskursfeld sind Artikel über Integrations- und Flüchtlingshelferinnen, wie auch Beiträge über erfolgreich integrierte Akademikerinnen, Unternehmerinnen oder Künstlerinnen mit „migrantischer“ Herkunft charakteristisch $^{8}$. Dem Thema der Partizipation migrantisch markierter Bürgerinnen, das im vorliegenden Beitrag analysiert wird, schenkt die Süddeutsche Zeitung selten Beachtung. Somit sind auch Artikel, in denen migrantisch markierte Bürgerinnen an den diskursiven Aushandlungen zum Gegenstand Migration/Integration als Sprecherinnen auftreten, im gesammelten Artikelpool selten.

Eine Sonderrolle nehmen die Jahre 2010 und 2011 ein, in denen in der Süddeutschen Zeitung eine mehrteilige Serie „Migranten in München“ publiziert wurde. Am 14. März 2011b lautet der Leadtext eines der Artikel: „München gilt als Vorzeigestadt in Sachen Integration - ist das tatsächlich so? In einer Serie lässt sueddeutsche.de Migranten und Experten zu Wort kommen.“, (SZ, 14. März 2011a). Publikationsanlass und Teil der Serie war die bereits erwähnte bundesweit vergleichende Studie zum Stand der Integration von Migrantinnen in München, die der Stadt und ihren Migrantinnen größtenteils eine hervorragende Integrationsfähigkeit bescheinigt (SZ, 14. März 2011b). Der zitierte Leadtext verweist mit Blick auf das Analysethema des Artikels auf dreierlei: Erstens wird München im Zeitungsdiskurs als urbane Integrationsgesellschaft bezeichnet, was sich von der in der postmigrantischen Perspektive geforderten An-

\footnotetext{
${ }^{7}$ https://www.mediadb.eu/forum/zeitungsportraets/ sueddeutsche-zeitung.html (letzter Zugriff: 23. Juni 2020).

${ }^{8} \mathrm{Ab} 2015$ tritt dieses Thema in der Süddeutschen Zeitung im Zusammenhang mit der wachsenden Ankunft Geflüchteter verstärkt auf.
}

erkennung der Bundesrepublik als Migrationsgesellschaft ${ }^{9}$ unterscheidet. Zweitens, dass die Süddeutsche Zeitung offensichtlich zur kritischen Auseinandersetzung mit der eigenen, mehrheitsgesellschaftlichen Position aus der „Perspektive der Migration" bereit ist. Drittens wird in dem Artikel eine fragwürdige Unterscheidung zwischen Expertinnen und Migrantinnen vorgenommen, die die Interpretation zulässt, dass „Migrantinnen“ nicht Expertinnen ihrer Situation sein können.

\section{Elemente des Diskurses}

Die Analyse des empirischen Materials in den folgenden Unterkapiteln wird, wie im 2. Kapitel beschrieben, entlang der vier zentralen Strukturelemente des Diskurses: (1) Gegenstand, (2) Begriffe, (3) Äußerungsmodalitäten und (4) Themen erfolgen. Meine Darstellung der Analyse werde ich entlang der zentralen Begriffe in den Artikeln: Ausländer, Heimat, Rassismus und Wandel strukturieren, die in den Artikeln in Verbindung mit dem Gegenstand des Diskurses Migration/Integration diskutiert werden. Kapitelweise werde ich die diskursiven Verschränkungen der Begriffe mit spezifischen Themen und Äußerungsmodalitäten in den Artikeln analysieren. Abschließend werde ich ausführlicher die diskursive Repräsentation und Konstruktion migrantisch markierter Sprecherinnen im Zeitungsdiskurs diskutieren, die ein zentrales Spezifikum der Äußerungsmodalitäten darstellen und wesentlich zur (De-)Legitimation der Sprecherinnen an den Aushandlungen in der Süddeutschen Zeitung beitragen.

\section{1 „Ausländische Bürger"}

Der Münchner Stadtrat:
Theodor Pregler (CSU) [...] betont mehrmals, dass das Gremium [Ausländerbeirat, Anmerkung der Autorin] nicht überflüssig sei. Er sehe aller- dings nicht die Notwendigkeit, dessen Kompeten- zen zu erweitern. Ein Budget brauche der Beirat nicht, denn für alles, was er mache, nämlich das in- ternationale Fest, zahle ohnehin die Stadt. [... ] Um den Beirat bekannter zu machen, könne nicht viel mehr getan werden [...]. Wahlen würden zu nichts führen, [...]. „Die Kontaktaufnahme zu ausländi- schen Bürgern ist nun einmal nicht ganz einfach" (SZ, 17. August 2016).

In dem Artikel werden die Auseinandersetzungen zwischen dem „Ausländerbeirat“ und den Stadträten in einem

\footnotetext{
${ }^{9}$ Der Begriff bezeichnet eine durch Migration geprägte Gesellschaft, die diese Tatsache für sich anerkennt (Canan und Foroutan, 2016:13) und stellt im Unterschied zur Einwanderungsgesellschaft eine allgemeinere Perspektive auf die gesellschaftlichen Veränderungen dar und berücksichtigt auch Migration als Gegenstand gewaltvoller politischer und alltagsweltlicher Aushandlungen (Broden und Mecheril, 2007:7).
} 
Münchner Viertel beschrieben. Dabei kommen beide Konfliktseiten und auch übergeordnete Gremien zu Wort. Wie im Zitat dargestellt, ist der zentrale Konfliktpunkt die Ablehnung weiterer Ressourcen für den Beirat von Seiten des Stadtrats, was mit den ,schwer erreichbaren ausländischen Bürgern", also einem defizitären Charakteristikum der Zielgruppe, begründet wird. Wie die Textpassage verdeutlicht, werden drei Aspekte von Seiten der Mehrheitsgesellschaft verhandelt: Fragen der Zugehörigkeit, der Partizipation und der Ressourcenverteilung, die hier zentrale Diskursthemen bilden. Der Begriff des „Ausländers“, der aus mehrheitsgesellschaftlicher Perspektive gesetzt wird (letztlich aber eine sehr heterogene Gruppe bezeichnet), klassifiziert den „Anderen“ auf der Grundlage seiner Nicht-Zugehörigkeit. Die Klassifikation knüpft an herkömmlichen Argumenten des mehrheitsgesellschaftlichen Negativdiskurses über migrantisch markierte Bürgerinnen an ${ }^{10}$, der auch in Form des Ghetto- und Parallelgesellschaftsdiskurses existiert (Wacquant, 2006; Ronneberger und Tsianos, 2009; Münch, 2010; Tsianos, 2013; Rodatz, 2014). Die Begriffssetzung legitimiert hier eine Grenzziehung, die eine mehrheitsgesellschaftlich definierte Inklusion geringen Umfangs zur Folge hat, die mit Einschränkungen in den Bereichen Partizipation (Kompetenzen und Wahlen) und Zugang zu Ressourcen (finanzielle und weitere Arbeitsmittel) einhergeht ${ }^{11}$. Die Reglementierung - hier des Wunsches nach mehr ehrenamtlichem Engagement und Partizipation am gesellschaftlichen Leben über den Zugang zu Ressourcen, ob ökonomischer, sozialer oder politischer Art, entfaltet im Alltag den nachhaltigen Effekt der sozialen Hierarchisierung von Gesellschaft und wird in der Literatur als struktureller Rassismus beschrieben (Balibar, 2005; Velho, 2010; Espahangizi et al., 2016). Diese Form der „Veranderung“ migrantisch markierter Bürgerinnen ist in der Süddeutschen Zeitung zwischen 2010 und 2016 weit verbreitet, z. B. in Artikeln über die schwer erreichbaren Schülerinnen oder das Bahnhofsviertel. Dieser Diskurs bildet das Gegenstück zum europäischen, modernen, rationalen, aktiven und eigenverantwortlich handelnden Menschen, wie er durch neoliberale Subjektivierungspraktiken hergestellt wird (Honneth, 2002; Rosol, 2013; Pütz und Rodatz, 2013). In meiner Artikelauswahl tritt der Negativdiskurs lediglich noch ein weiteres Mal, im Artikel über Amir Roughani auf:

„Ich kam in eine Ausländerklasse“, sagt Roughani, ,aber das ging nicht gut bei mir, denn da habe ich nur Türkisch gelernt“. [...] Roughani zeigt auf seine Narben. Ausländer haben sie ihm damals zugefügt, sagt er, [...] (SZ, 14. März 2011c).

\footnotetext{
${ }^{10}$ Prominentes Beispiel dafür sind beispielsweise die Publikationen Thilo Sarrazins.

${ }^{11}$ Wie im Artikel deutlich wird, ist die finanzielle Unterstützung und die dadurch mögliche Erweiterung der ehrenamtlichen Aufgaben der Beiräte in anderen Vierteln Münchens durchaus üblich.
}

Auch Amir Roughani liegt dem Staat zunächst auf der Tasche. Er wohnt im Kinderheim, kann kein Wort Deutsch [...]. Doch er beißt sich durch. [...] lernt Deutsch, freundet sich mit einem Deutschen an, [...] (ebd.).

In dem Artikel, in dem Roughani aus Anlass seiner Auszeichnung 2011 mit dem Münchner Phönixpreis für „Unternehmerinnen mit Migrationshintergrund" 12 vorgestellt wird, dient sein Lebenslauf als Paradebeispiel für erfolgreiche Integration im Sinne des neoliberalen Subjekt- und auch des neueren bundesdeutschen Integrationsparadigmas, dass u. a. die „migrantischen“ ökonomischen Potentiale in den Vordergrund rückt (Lanz, 2009; Pütz und Rodatz, 2013). Roughani stellt sich selbst und seinen Erfolg als Verdienst und Ergebnis seines Fleißes, seiner aktiven Integrationsbemühungen, seiner Anpassungsbereitschaft und Bescheidenheit dar. Davon zeugen die zahlreichen Verben im Text: „er geht“, „besteht“, „beginnt“, „hängt sich rein“, „,finanziert sich mit Nebenjobs“, „,bittet“, ,,organisiert“, „,macht sein eigenes Ding: Vispiron“ (ebd.). Dabei benutzt er den Begriff des Ausländers als Abgrenzungsfolie und verknüpft es mit dem Thema Integration. Indem er ,andere Migrantinnen“ als Gegenbild zu seiner eigenen Geschichte setzt, rechtfertigt er ein System, das zwischen verdienter und nicht-verdienter Inklusion differenziert. Ausgehend von der journalistischen Rahmung seiner Sprechakte finden seine Aussagen im Artikel öffentlich Unterstützung und Zustimmung. Allerdings verweisen die Schilderungen auch darauf, welche hohen Anforderungen an die Aufnahme und Akzeptanz in der bundesrepublikanischen Gesellschaft letztlich geknüpft sind. $\mathrm{Zu}$ Sarrazins umstrittenen Analysen und Forderungen zum Thema Integration ${ }^{13}$ befragt:

,[...] es gibt vieles worauf er [Sarrazin, Anmerkung der Autorin] zu recht [!] hinweist“, sagt er. Das Problem sei doch, dass in Deutschland bis heute keine ernsthafte Integrationspolitik betrieben werde. Es müsse besser gefiltert werden. Und dann: Fördern und Fordern. „Man muss den Migranten klare Regeln aufzeigen“ (ebd.).

Auf der Grundlage seiner Zeugenschaft, wenn auch mit Abstrichen, bestätigt er Sarrazins Kritik an den ,schlechten Ausländern“"14 mit dem Nebeneffekt, dass er sie aus der

\footnotetext{
${ }^{12} \mathrm{Mehr}$ dazu auf https://www.muenchen.de/rathaus/ Stadtverwaltung/Referat-fuer-Arbeit-und-Wirtschaft/

Arbeitsmarktpolitik/Kompetenzentwicklung/Phoenix.html, (letzter Zugriff: 27. Februar 2019).

${ }^{13}$ Es geht dabei um Sarrazins 2010 erschienenes Buch „Deutschland schafft sich ab“. Darin beschreibt er u. a. die Gefahr des demografischen Wachstums in bildungsfernen Schichten und charakterisiert Muslime als erblich bedingt bildungsfern und schwer integrierbar.

${ }^{14}$ Inwiefern dies als Folge der Internalisierung mehrheitsgesellschaftlicher Denk- und Subjektivierungsweisen interpretiert werden
} 
Perspektive der Migration legitimiert und damit letztlich zur Normalisierung von Sarrazins Position beiträgt.

In beiden Artikeln sind die Themen Integration und der Negativdiskurs über migrantisch markierte Bürgerinnen, hier als „Ausländer“ bezeichnet, in Verschränkung mit den Themen Partizipation und Ressourcen dominant. Sie dienen in beiden Fällen der Differenzierung der Zugehörigkeit und rechtfertigen eine Einschränkung der Partizipationsmöglichkeiten. Im zweiten Artikel wird diese Matrix der Grenzziehungen und Hierarchisierung von Gesellschaft mit Verweis auf den zu leistenden Beitrag vervollständigt. Mit Blick auf die Sprecherinnenpositionen in den Artikeln und das sich darin wiederspiegelnde Verhältnis zwischen Mehrheitsgesellschaft und Minorisierten ist in beiden Fällen die spezifische Rolle der journalistischen Rahmung interessant. Im ersten Artikel wird die konservative Position des Stadtrats journalistisch genutzt, um den Bogen vom Argument der „schwer erreichbaren ausländischen Bürger" zur Kritik am Stadtrat, einer Institution der Mehrheitsgesellschaft, aufzuspannen und seine auf Ausschluss zielende Position kritisch zur Debatte zu stellen. Demzufolge wird die kritische Perspektive im ersten Fall als ein Blick der Mehrheitsgesellschaft auf sich selbst gerahmt und im zweiten als der Blick der Minorisierten auf sich selbst. In beiden Artikeln wird die Perspektive der Migration auf den Gegenstand Migration/Integration im Zeitungsdiskurs präsent. Im ersten findet sie journalistische Unterstützung, im zweiten spielt auch der Aspekt der Ausnutzung zur Legitimation der Grenzziehungen eine Rolle. Die Kämpfe der Migration, die in diesen Artikeln zur Sprache kommen, sind (1) die Auseinandersetzung mit den negativen mehrheitsgesellschaftlichen Zuschreibungen, für deren Wirkmächtigkeit ihre Legitimation im zweiten Artikel steht, (2) die Abhängigkeit von mehrheitsgesellschaftlichen Ressourcen, und (3) am Beispiel Roughanis die hohen Ansprüche der mehrheitsgesellschaftlichen Anerkennung.

\section{2 „Zweite, neue Heimat"}

Die 48-jährige Ribas sagt: ,In der Schreibwerkstatt merken die Migrantinnen, dass sie was zu sagen haben." [...] Geschichten die in keinem Integrationsbericht der Regierung vorkommen und in keiner Zeitung nachzulesen sind. Calda sagt: ,Wir haben erkannt, dass wir uns selber zu Wort melden müssen“ (SZ, 17. August 2010).

In der Reportage Schreibwerkstatt erzählen migrantisch markierte Frauen von ihren Begegnungen und Alltagserfahrungen mit der bundesrepublikanischen Gesellschaft. In vielen Punkten sprechen sie erlebte Probleme, Missverständnisse und Ausgrenzungen aus ihrer Perspektive (der Migrati-

kann oder als Folge der Einsicht, dass Kritik an der Mehrheit von Seiten Minorisierter nicht in jedem Fall erwünscht ist, ist an dieser Stelle zweitrangig. on) an, formulieren eigene Interpretationen der erlebten gesellschaftlichen Wirklichkeit. Die von ihnen in die diskursiven Aushandlungen eingebrachten Themen umfassen unterschiedliche Formen der differentiellen Inklusion, denen sie u. a. mit eigenen Interpretationen zentraler Begriffe wie Integration und Heimat entgegentreten.

„Meinen ursprünglichen Beruf habe ich hier [...] nicht ausüben können. [...] der Mangel an Bereitschaft [...] außerhalb Deutschland[s] erworbene Qualifikationen anzuerkennen [...]. In der Verzweiflung nehmen viele [...] niedrige Jobs an. Meistens sind diese [...] zeitlich sehr ungünstig gelegen für ein Familienleben und die Partizipation am gesellschaftlichen Leben. Später wundert man sich, dass noch so viele Zuwanderer sich mit der Integration schwer tun!“" (ebd.).

Auf der Grundlage persönlicher Lebenserfahrungen werden hier die ökonomischen Grundlagen des Systems der differentiellen Inklusion und ihre Auswirkungen im Alltag als Fortschreibung einer fragmentierten, unvollständigen Integration beschrieben.

Das 2. Thema bildet die gängige Konzeptualisierung von Heimat, deren mehrheitsgesellschaftlich verbreitetes essentialistisches Verständnis eine weitere Form darstellt, eine strategisch selektive Inklusion zu legitimieren. Dem setzen die Frauen eine nicht lokal gebundene, sondern praxeologisch und emotional orientierte Lesart ${ }^{15}$ entgegen:

„Ich bin glücklich in meiner zweiten, neuen Heimat, die ich lieben gelernt habe [...]. Ich sehe es als ein großes Privileg, zwei ,Heimaten` zu haben $[\ldots]^{\text {“ }}$ (ebd.).

Sie begreifen Heimat als soziale Praxis des Miteinanders und auch als Ergebnis eines mehrheitsgesellschaftlichen Handelns:

Sarra Chaouch, 23, die in München geboren ist [...]. „Die Bayern sind besonders stolz auf ihre Traditionen, [...]. Aber sie müssen mich auch akzeptieren. Ich würde München noch viel mehr lieben, wenn man mir das Gefühl gäbe, das ist meine Heimat“" (SZ, 4. Juli 2016; vergleiche auch SZ, 22. November 2016).

Diese eigene Interpretation der Wirklichkeit, die die Frauen in die Aushandlungen einbringen, sind Teil der diskursiven „Kämpfe der Migration“. Sie basieren einerseits auf ihrer Erkenntnis, sich nicht auf Fremdbeschreibungen reduzieren lassen zu wollen (Scheel, 2015) und andererseits auf der kritischen Auseinandersetzung mit mehrheitsgesellschaftlichen Argumentationen. Eine besondere Bedingung ihrer Kritik ist,

\footnotetext{
${ }^{15}$ Mehr zur kritischen Debatte um den Heimatbegriff siehe bei Binder (2010).
} 
dass sie diese aus dem ihnen journalistisch bereitgestellten Raum des öffentlichen Zeitungsdiskurses heraus formulieren. Somit operieren sie aus dem Raum des „Anderen“ heraus (De Certeau, 1988). Dabei arbeiten sie mit Formen der Re-Interpretation und der „Umleitung“ von Kritik, aber auch der Aneignung mehrheitsgesellschaftlich relevanter Begriffe und der Erweiterung ihrer Bedeutungen in einem inklusiven Sinne. Jedoch führen sie diese diskursiven Aushandlungen öffentlich und stellen ihre Erfahrungen mit dem nicht für alle sichtbaren System der Grenzziehungen zur Diskussion, z. B. die Grenzen der Anerkennung oder des partiellen sozialen Einschlusses durch spezifische Arbeitsverhältnisse (Mezzadra und Neilson, 2013), die ihren Alltag durchziehen und als mehrheitsgesellschaftlich machtvoll strukturierten Raum erscheinen lassen. Damit fordern sie die Mehrheit zu einer Reflektion der Wirklichkeit aus ihrer Perspektive (der Migration) auf. Gleichzeitig formulieren sie damit ihr Recht auf Anerkennung, auf soziale und ökonomische Partizipation und nehmen sich das Recht, die Gesellschaft diskursiv mitzugestalten.

\section{3 „Kleine Apartheid"}

In meiner hier zugrunde liegenden Artikelauswahl machen migrantisch minorisierte Bürgerinnen auch Fälle rassistischer Praktiken, die sie in ihrem Alltag erleben, zum Thema ihrer diskursiven Aushandlungen. Dazu gehören strukturelle und gleichermaßen alltägliche Rassismuserfahrungen ${ }^{16}$. Im Jahr 2016 weisen diese Berichte in der Süddeutschen Zeitung eine gewisse Zunahme auf. Das Auftreten dieser Fälle ist allerdings nicht ausschließlich auf aktuelle Konjunkturen zurückzuführen, denn „Gastarbeiterkinder“, wie der Künstler Tuncay Acar und der Lebensmittelhändler Serdar Yildirim, berichten u. a. von ihren Jahrzehnte zurückliegenden Kindheitserlebnissen (SZ, 14. März 2011a, 12. Januar 2016).

In dem Interview, das mit Yildirim anlässlich der Verleihung des Phönixpreises 2015 geführt wird, wird von dem Einzug der ersten türkischen Handelsfirma 1983 in die Münchner Großmarkthalle berichtet:

[...] es gab Probleme, im Grunde waren es die gleichen, die Serdar Yildirim als Kind [... ] erlebte. Es gab Anfeindungen, Rempeleien und Handgreiflichkeiten. Es gab Rassismus. Oder wie soll man es nennen? „Kleine Apartheid“, [...] (SZ, 12. Januar 2016).

Auch im Artikel über und mit Sükrü Keser, Mitbegründer und Kandidat der Aktiven Internationalen Jugendliste für die Wahl des Münchner Ausländerbeirats, wird berichtet:

[...]immer wieder fühlt sich der Pädagoge [...] diskriminiert. Bei seinem ersten Vorstel-

\footnotetext{
${ }^{16}$ In den Artikeln spielt auch die positiv ,verbesondernde“ Form des Rassismus eine Rolle, auf die ich in meinen Analysen allerdings nicht weiter eingehen kann.
}

lungsgespräch sei er als erstes gefragt worden: „Sind Sie Türke?“ Als er das bejahte, hieß es: „Dann haben wir leider nichts für sie“ (SZ, 10. Dezember 2010).

Beide Erfahrungen sind Beispiele für den in der Gesellschaft strukturell verankerten Rassismus und beschreiben die Limitierung des Zugangs zu ökonomischen Ressourcen und somit die erlebte differentielle Inklusion, die über die Konstruktion der „Andersheit“" von Seiten der Mehrheitsgesellschaft legitimiert wird ${ }^{17}$. Im ersten Fall wird der Aspekt der Verteidigung der Vormachtstellung der mehrheitsgesellschaftlichen Händlerinnen auf dem Großmarkt besonders deutlich. Die Verschränkung von ethnischer Essentialisierung mit ökonomischer Differenzierung gilt als zentrale Artikulationsweise kulturrassistischer Ordnungen (Geulen, 2007; Schrödter, 2007; Broden und Mecheril, 2010).

Andere migrantisch markierte Bürgerinnen berichten über ihre Erfahrungen mit Formen des alltäglichen, anonymen Alltagsrassismus ${ }^{18}$ im öffentlichen Raum:

[...] es sind nicht nur Worte, die verletzen. „Die Blicke spürt man“, sagt Keser. „Auf der Straße, im Bus - ständig“ (SZ, 10. Dezember 2010; vergleiche auch SZ, 4. Juli 2016).

Oder:

Im September schlug ein Mann in der Münchner U-Bahn einer Muslima zwei Mal ins Gesicht, beleidigte sie und ihre Mutter und schrie, sie sollten Deutschland verlassen (SZ, 22. November 2016). ,[...] in der U-Bahn [...] kommen Sprüche wie ,Allahu akbar' oder ,Jetzt geht die Bombe hoch““ (SZ, 4. Juli 2016).

Die Beispiele verweisen zum einen auf die große Bandbreite der Erfahrungen, und zum anderen auf eine differenzierende rassistische Praxis gegenüber migrantisch markierten Frauen und Männern. Sie widerspiegeln auch die in einigen Analysen beschriebene Konjunktur der semantischen Verknüpfung von Islam, Terror und Kopftuch als Verweis auf Rückständigkeit und Integrationsunwilligkeit (Jäger, 2010; Spielhaus, 2018).

Kennzeichnend für die Darstellungen der rassistischen Erfahrungen in den Artikeln sind die dafür verwendeten

\footnotetext{
${ }^{17}$ Die ethnisch essentialisierende Form der Konstruktion von „Andersheit“ wird besonders im Fall Kesers deutlich, dessen pädagogische Bildungslaufbahn an der Münchner LMU erfolgte (SZ, 10. Dezember 2010).

${ }^{18}$ Die verbreitete Erklärung, dass es sich dabei um „tragische“ Einzelfälle von Seiten de-privilegierter Angehöriger der Mehrheitsgesellschaft handelt, greift zu kurz (Broden und Mecheril, 2010). Wie Butler (2006:81) darlegt, wird ,,das Subjekt, das gesellschaftlich verletzende Worte äußert, von einer vorangegangenen Kette verletzender Anrufungen mobilisiert", somit sind auch alltagsrassistische Äußerungen als Artikulationen einer rassistischen Ordnung anzusehen.
} 
Begrifflichkeiten. Die migrantisch markierten Bürgerinnen nutzen, mit Ausnahme des Lebensmittelhändlers Yildirim, durchweg Ersatz- bzw. umschreibende Begriffe. Sie sprechen beispielsweise von Erfahrungen mit Ausgrenzung (SZ, 14. Juli 2016), von wachsender Diskriminierung (SZ, 22. November 2016), von Unverschämtheiten und respektlosem Umgang (SZ, 14. März 2011). In diesen Artikeln wird der Rassismusbegriff teilweise von journalistischer Seite zur Einordnung der Vorfälle benutzt (SZ, 14. Juli 2016 und 22. November 2016). Dies kann einerseits als Ergebnis einer neuen Sensibilisierung innerhalb der Mehrheitsgesellschaft betrachtet werden, die u. a. auf die verstärkten Auseinandersetzungen mit der ,european ideology of racelessness“ (El-Tayab, 2011) zurückzuführen ist, und zu denen migrantisch markierte Wissenschaftlerinnen ihren Beitrag migrantischer Kämpfe geleistet haben. Andererseits widerspiegelt dieses Verhältnis der Äußerungsmodalitäten auch die anhaltenden Schwierigkeiten, Rassismus offen $\mathrm{zu}$ thematisieren, insbesondere von Seiten Betroffener. In jedem Fall belegen die Vorfälle deutlich, dass der Alltag und der öffentliche Raum aus migrantischer Perspektive mitunter auch ein feindliches Feld darstellen, in denen Formen der körperlichen und symbolischen Gewalt die sozialen, ökonomischen und politischen Grenzziehungen begleiten. Somit stellt Rassismus ein wichtiges Diskurselement dar, das die diskursiven Beziehungen zwischen Mehrheitsgesellschaft und Minorisierten organisiert, was diesen als wichtigen Begriff der Reflektion und Analyse dieses Verhältnisses notwendig macht.

Aus den Artikeln geht auch hervor, dass migrantisch markierte Bürgerinnen sich im gemeinsamen Austausch, organisiert in Selbsthilfegruppen, auch in Koalition mit Vertreterinnen der Mehrheitsgesellschaft diesen Vorfällen stellen. Dabei formulieren sie Forderungen nach Gleichberechtigung und - wie im Falle Sükrü Kesers - den Wunsch, politisch aktiv zu werden (SZ, 10. Dezember 2010). Aus der Perspektive der „Kämpfe der Migration“ findet, wenn auch verstreut, eine organisierte Auseinandersetzung mit Rassismus statt, die gegenseitige Solidarisierungen und Selbstermächtigung ermöglicht, neue Selbstbilder und -praxen erzeugt (Broden und Mecheril, 2010:17).

\section{4 „Meister der Integration“}

In einigen Artikeln meiner Auswahl aus der Süddeutschen Zeitung wird der Beitrag migrantisch markierter Bürgerinnen im Stadtalltag zum Thema der diskursiven Aushandlungen. Beispielsweise sagt Yildirm in dem bereits zitierten Interview auch:

Seine eigene Herkunft spielt heute in der Großmarkthalle keine Rolle mehr. „Die deutschen Händler sind längst nicht mehr feindlich gesinnt“, [...]. Er lächelt. „Heute sind sie froh, dass wir da sind - ohne den türkischen Einzelhandel wäre die Großmarkthalle nicht mehr lebensfähig“" (SZ, 12. Januar 2016).

Damit verweist er auf seinen mehrheitsgesellschaftlich anerkannten Beitrag zur Münchner Ökonomie, in diesem Fall zum Erhalt der Großmarkthalle und damit auch zum Fortbestand mehrheitsgesellschaftlicher lokaler Unternehmen, was seine Inklusion in die Gemeinschaft der Händler zur Folge hatte. Wenn man seine Sätze aus einer Ursache-WirkungLogik liest, dann lautet die Aussage: Weil er ökonomisch inklusiv und nicht verdrängend wirkte, haben sich die mehrheitsgesellschaftlichen Praktiken der Grenzziehung (Kapitel 5.3) in Inklusion gewandelt. Somit ist die mehrheitsgesellschaftliche Öffnung als eine Folge seiner Arbeitsweise zu betrachten, seiner Beharrlichkeit und Nachsicht - die durchaus als mikropolitische Praktiken migrantischer Kämpfe gelten können (Marche, 2012) - gegenüber mehrheitsgesellschaftlichen Ausschlusspraktiken.

In einem anderen Artikel wird aus der mehrheitsgesellschaftlichen Perspektive über den Leiter eines Münchner Jugendzentrums, Deniz Dadli, im Zusammenhang mit den positiven Veränderungen im Stadtteil berichtet. Der „Deutschtürke“ wird als „Meister der Integration“ bezeichnet:

[...] dass Dadli durch seine Herkunft das Vertrauen türkischer Eltern gewonnen habe - und viele migrantische Kinder in die Freizeitstätte geholt habe. [...] Mal wieder reden Oberschleißheimer miteinander. Sein Jugendzentrum ist längst das Kulturzentrum der Stadt geworden (SZ, 2. November 2015).

Auch hier ist das zentrale Thema der Beitrag eines eigentlich migrantisch markierten Bürgers zu den Veränderungen im Stadtteil, die gleich mehrere innergesellschaftliche Fragmentierungslinien betreffen - zwischen den Generationen, zwischen Mehrheit und Migrantisierten sowie zwischen Zentrum und Randvierte ${ }^{19}$. Sein Beitrag und der davon ausgehende Wandel wird diskursiv auf verschiedene Weise entworfen: „,versammeln“, ,Vielfalt“, „,Vertrauen gewinnen“, „hineinholen“ und „miteinander reden“. Diese Auflistung offenbart dreierlei: erstens den Beitrag migrantisch markierter Bürgerinnen zu den gesellschaftlichen Veränderungen und zweitens, dass es für diesen Wandel offensichtlich noch keinen übergeordneten Begriff gibt, denn die Veränderungen werden im Detail aufgezählt. Insgesamt wird in diesem Artikel jedoch ein begrifflicher Wandel vollzogen, der in meiner Auswahl alleinstellend ist: Es wird nicht, wie in anderen Artikeln, von „Ausländern“ gesprochen. Deniz Dadlis Mehrfachzugehörigkeit wird mit der Bezeichnung

\footnotetext{
${ }^{19}$ Durch sein Engagement trägt Dadli auch zu einer Reskalierung zwischen der globalen Welt und dem Münchner Stadtteil bei, indem er sich gemeinsam mit den Bewohnerinnen sozial in unterschiedlichen Krisen- und Kriegsgebieten engagiert und sie sich mit den globalen Zusammenhängen auseinandersetzen.
} 
des „Deutschtürken“ anerkannt und er in die Mehrheitsgesellschaft aufgenommen. Der in beiden Artikeln beschriebene mehrheitsgesellschaftliche Wandel gegenüber migrantisch Minorisierten fand in der Arbeitswelt statt und ist Ergebnis ihrer Leistungen, ihrer inklusiven Praktiken und ihres Beharrungsvermögens gegenüber diversen Grenzziehungen. Dieser leistungsorientierte Blick auf Inklusion, der auch bereits in Kapitel 5.1 in diesem Beitrag diskutiert wurde, basiert auf einem gängigen (neorassistischem) Muster der Differenzierung zwischen guten und schlechten Migrantinnen (Lanz, 2007; Pütz und Rodatz, 2013; Riedner, 2018).

Dieses selektive Inklusionsregime wird von migrantisch markierten Bürgerinnen wiederum kritisch in die diskursiven Aushandlungen in der Süddeutschen eingebracht. Der bereits zitierte Tuncay Acar sagt dazu:

Man kann nicht sagen, nur die Mezut Özils sind willkommen, die anderen nicht (SZ, 14. März 2011a).

Den Kontrapunkt zu diesem mehrheitsgesellschaftlichen leistungsorientierten Blick auf Inklusion bildet die ,passive (nicht leistungsorientierte) Migrantin“ und neuerdings, wie einige Aussagen migrantisch markierter Bürgerinnen in den Artikeln verdeutlichen, die muslimische Migrantin:

,[...] wenn Parteiplakate [der AfD, Anmerkung der Autorin] suggerieren: Deutschland ist inkompatibel mit dem Islam. So wird ein Teil der deutschen Gesellschaft einfach ausgeklammert" (SZ, 22. November 2016).

Die zunehmende semantische Verschränkung von Migration und Islam (Spielhaus, 2018) bietet einen neuen Referenzpunkt zur Fortschreibung des Systems der differentiellen Inklusion.

Der im Beitrag bereits mehrfach zitierte Sükrü Keser kritisiert die Differenzierung zwischen einerseits den ökonomischen Anforderungen an migrantisch markierte Bürgerinnen und andererseits ihrem reglementierten Zugang zu Bildung, sozialen sowie politischen Ressourcen und fordert Gleichberechtigung:

„Wir sind auch ein Teil der Gesellschaft.“ Dass er Lösungen für Münchens Verkehrskonzepte suchen [...], aber nicht den Oberbürgermeister und Stadtrat wählen darf, [...] (SZ, 23. Februar 2016).

Die Internationale Jugendliste kämpft gegen das dreigliederige Bildungssystem, weil es vielen keine Chance lasse, insbesondere den Migranten nicht. [und dafür, Anmerkung der Autorin] dass alle Studenten, egal welcher Herkunft, Bafög beantragen können, das Mehrsprachigkeit als zusätzliche Qualifikation anerkannt wird [... ] (SZ, 10. Dezember 2010).
Damit führt er einige Aspekte des Systems der differentiellen Inklusion an, das über zahlreiche Momente der Kontrolle den Zugang zu universellen, politischen Rechten und sozialen Ressourcen limitiert, und die Alltage vieler migrantisch minorisierter Bürgerinnen als ein komplexes Arrangement ineinander verschachtelter Ein- und Ausschlüsse nachhaltig prägt (Mezzadra und Neilson, 2013). Seine Forderung positioniert das Thema Teilhabe und Partizipation somit als alltägliches Feld migrantischer Kämpfe.

Im Zeitungsdiskurs wird sowohl aus der Perspektive der Migration ebenso wie der Mehrheitsgesellschaft eine Öffnung in partikulären Alltagskontexten wahrgenommen. Diese weist jedoch eine leistungsorientierte Prägung auf und bezieht sich nicht auf die zwischenmenschliche, affektive Ebe$\mathrm{ne}^{20}$. Somit ist dieser Wandel, die Anerkennung der Leistung, Bestandteil des komplexen Systems der differentiellen Inklusion. Auch der im Diskurs fehlende Überbegriff für den Wandel spricht für das anhaltende „Hadern der Gesellschaft mit Migration“ (Spielhaus, 2018) und zeugt von der komplexen Verschränkung von partiellen Öffnungen und neuen Grenzziehungen ${ }^{21}$. Der journalistische Diskurs lässt der pluralen Gemengelage der Positionen Raum zur Artikulation, ohne jedoch die Widersprüchlichkeit bzw. die daraus resultierenden Konsequenzen zur Debatte zu stellen. Diese diskursive Praxis trägt, wie auch von Jäger (2010) beobachtet, letztlich weniger zu einer Normalisierung von Migration bei, sondern zur Breite und eben auch Gegensätzlichkeit der Positionen zum Thema.

\section{5 „Quotentürken“ und „Bio-Deutsche“}

Mit dem diskursanalytischen Element der Äußerungsmodalitäten sind verschiedene journalistische Formen der Subjektkonstruktion $^{22}$ eng verbunden. Sie besitzen eine besondere Relevanz im Prozess der (De-)Legitimierung migrantisch markierter Bürgerinnen. Daher soll diesem Element der Organisation von Aussagen eine besondere Aufmerksamkeit an dieser Stelle gelten. In meiner Artikelauswahl konnte ich drei zentrale Formen herausarbeiten, die das journalistische Sprechen in unterschiedliche Beziehungen zum migrantisch markierten Sprechen setzen und für unterschiedliche Formen der Subjektkonstruktion migrantisch markierter Gesprächspartnerinnen stehen.

Für die erste Form der Beziehung zwischen journalistischem und migrantisch markiertem Sprechen steht das In-

\footnotetext{
${ }^{20}$ Bei Schrödter (2007) mehr zu den Ebenen der Anerkennung und Bedeutung der fehlenden Anerkennung auf affektiver Ebene.

${ }^{21}$ Zum Kontinuum zwischen Negativ- und Potentialdiskursen über Migrantinnen in integrationspolitischen Dokumenten und Praktiken vergleiche auch Pütz und Rodatz (2013).

${ }^{22}$ Ich spreche hier von diskursiven, genau genommen fragmentierten Strategien der Subjektkonstruktion, anstatt von Repräsentationen, da eine Vorstellung von den Gesprächspartnerinnen selektiv über einzelne, strategisch gesetzte Beschreibungen hervorgerufen wird.
} 
terview mit Tuncay Acar. Das Interview ist mit Blick auf die Erzählstruktur alleinstellend. Es besteht aus kurzen Fragen und ausführlichen Antworten und gibt dem Künstler Raum, die Leserinnen an seinen Sichtweisen und Kritiken teilhaben zu lassen. Sein Sprechen ist selbstbewusst, offen, provozierend:

„Allerdings kenne ich viele Bio-Deutsche..." sueddeutsche.de: „Bio-Deutsche?“ Acar: „Das ist meine Antwort auf den Ausdruck Migrationshintergrund, Deutsche ohne migrantische Vergangenheit" (SZ, 14. März 2011a, Auslassungen im Original).

und

Bedingungen setzend: „Bitte nicht die Quotentürkennummer draus machen. Da werd ich sauer" (ebd.).

Aufbegehrend, rebellisch spricht er die Mehrheitsgesellschaft im Raum des mehrheitsgesellschaftlichen Zeitungsdiskurses (im Raum des „Anderen“ nach de Certeau) an, eignet sich diesen an, indem er mit eigenen Begriffssetzungen die sozialen Beziehungen zueinander in diesem Raum neu ordnet. Zusätzlich knüpft er sein Sprechen an Bedingungen und reklamiert Entscheidungsmacht für sich. Dadurch entsteht auch der Gesamteindruck, dass er die Spielregeln in diesem Raum umkehrt und in seinem Sinne setzt. Er benutzt den ihm journalistisch gebotenen Rahmen und schafft sich gegenüber der Journalistin eine gleichwertige Sprechposition.

Für die zweite Form der Sprecherinnenbeziehung zwischen Mehrheit und Minorisierten steht das Interview mit dem Unternehmer Roughani. In diesem wird hauptsächlich aus der journalistischen Position seine Lebenserzählung zusammengefasst wiedergegeben. Roughani kommt nur selten im direkten Zitat zu Wort. Im Artikel wird seine Lebensgeschichte letztlich als Kommentar zu Sarrazins Thesen genutzt:

Was hält so jemand von Sarrazins Thesen? Gar nicht mal so wenig (SZ, 14. März 2011),

erzählt der Journalist den Leserinnen. Insgesamt stellt sich die Frage, inwieweit Roughani aus eigener Motivation spricht oder die ihm bekannten mehrheitsgesellschaftlichen Erwartungen bedient oder inwiefern er von journalistischer Seite zur Illustration und dadurch auch zur Normalisierung einer zwischen guten und schlechten „Ausländerinnen“ differenzierenden Diskursposition benutzt wird ${ }^{23}$.

Neben den beiden dargestellten Formen tritt in der Auswahl der zehn analysierten Artikel zwischen 2010 und 2016 in der Süddeutschen Zeitung eine dritte Form auf, das gemeinsame Sprechen über Migration, das am weitesten verbreitet ist. Dafür ist wiederum der Artikel über Sükrü Keser,

\footnotetext{
${ }^{23}$ Was dem Journalisten die Möglichkeit eröffnet, nicht selber darüber urteilen zu müssen.
}

dem Kandidaten der Internationalen Jugendliste für die Wahl des Münchner Ausländerbeirats, ein gutes Beispiel. Die Journalistin führt zwar sprechend durch den Beitrag, lässt Keser jedoch in zahlreichen Zitaten direkt zu Wort kommen:
„Auch nach 60 Jahren Migrationsgeschichte gibt es noch jede Menge Vorurteile." Nun will er dies nicht mehr hinnehmen. [...] Er fordert, dass Mi- granten ohne deutschen Pass künftig auch bei kom- munalen Wahlen ihre Stimme abgeben dürfen. „Christian Ude ist schließlich auch unser Bürger- meister“, [...] (SZ, 10. Dezember 2010).

Diese verwobene Erzählstruktur, die ich als ein gemeinsames Sprechen zwischen Zeitungsdiskurs und migrantisch markierten Bürgerinnen interpretiere, besteht darin, dass im Artikel seine Themen aufgegriffen, ihm Raum für seine Aussagen gegeben und er unterstützend kommentiert wird. Charakteristisch dafür ist beispielsweise auch die journalistische Unterstützung seines politischen Engagements, seiner Erfahrungen mit Diskriminierung und Gefühle der Wut:

haut mit der Faust auf die Tischplatte, mit der anderen drückt er den Deckel seiner Cola-Flasche ein. Der 34-Jährige ist wütend. [... ] fühlt sich [...] diskriminiert (SZ, 10. Dezember 2010).

Genau genommen sind diese Beschreibungen gesellschaftlich durchaus umstritten, über Gefühlsausbrüche können Sprecherinnen diskursiv leicht delegitimiert werden; inwiefern Wut beispielsweise als positive und produktive gesellschaftliche Kraft und nicht als zerstörerische Emotion interpretiert wird, hängt von der journalistischen Rahmung und letztlich von der Leserin ab. In der Sprechkonstellation im Artikel wird die Situation journalistisch positiv kommentiert und somit institutionell legitimiert ${ }^{24}$.

Alle drei Formen der Sprecherinnenkoalitionen machen die Wirkmächtigkeit der journalistischen Rahmung für die Rezeption der Aussagen deutlich. Ähnlich ist die Bedeutung der journalistischen Konstruktion der Sprecherinnen zu bewerten, die sich komplementär, ausgleichend oder kontrapunktisch zu der jeweiligen Erzählstruktur verhalten kann.

Die 1. Strategie wird in vielen Artikeln genutzt. Die Sprecherinnen werden mit Namen und Herkunftsländern adressiert, wodurch eine eindeutige ethnische Markierung, eine Zementierung ihres migrantischen Status und nachhaltige Unterscheidung von mehrheitsgesellschaftlichen Sprecherinnen erfolgt. Ein Beispiel dafür bietet auch das Interview mit Tuncay Acar aus der Serie „Migranten in München“, die, wie eingangs schon erwähnt, „Migranten“ und „Experten“ befragt. Im Artikel wird er als „kreativer Kopf“", Künstler und

\footnotetext{
${ }^{24}$ Eine Analyse hinsichtlich der Teilnahme migrantisch markierter Bürgerinnen als Journalistinnen am Zeitungsdiskurs kann ich auf der Grundlage meines Materials nicht vornehmen. Denn m. E. ist allein die Tatsache, ob ein Name deutsch klingt oder nicht, für einen substantiellen Rückschluss auf migrantische Erfahrung, Markierung oder Positionierung nicht ausreichend.
} 
Kulturschaffender sowie Mitbegründer des Veranstaltungsorts Import-Export-Bar eingeführt, aber auch als:

Sein Deutsch ist akzentfrei. [...] Doch der Name verrät es: Tuncay Acar ist Sohn türkischer Gastarbeiter. (SZ, 14. März 2011a)

vorgestellt. Das wirft u. a. die Frage auf, in welcher Funktion Acar für die Süddeutsche Zeitung und ihre Leserinnen spricht. Der erste Teil der Aufzählung legt einen Expertenstatus nahe, während der zweite Teil ihn eindeutig migrantisiert. Die Wirkung des Ausdrucks ,verräterischer Name“ ist nicht zu unterschätzen, denn der Sprechakt verweist auf die spezifische diskursive Ordnung, in der niemand aus dem migrantischen Status entlassen wird.

Die 2. Strategie arbeitet mit punktuellen Verweisen auf symbolisch hochgradig aufgeladene Embleme: Signalwörter (z. B. Muslima) oder spezifische Lebensstilelemente (Kleidungsstücke, Haar- oder Bartschnitt). Diese bieten den Vorteil einer auf Homogenisierungen beruhenden Erkennbarkeit, der semantische Kurzschlüsse zugrunde liegen. In meiner Artikelauswahl lassen sich zwei Umgangsweisen mit solchen emblematischen Elementen beobachten: Die ,verandernde“ Form:

eine Frau Mitte 40, die sich erst vor einigen Jahren dafür entschied, als Ausdruck ihres Glaubens ein Kopftuch zu tragen. (SZ, 22. November 2016)

und die inkludierende Form:

Keser hat eine Hornbrille auf, sein Bart ist an der Oberlippe und am Kinn gestutzt. Er trägt ein Holzfällerhemd und darüber einen dicken braunen Wollpulli (SZ, 10. Dezember 2010).

Während im ersten Beispiel die Frau durch das Kopftuch von der Mehrheitsgesellschaft distinguiert wird ${ }^{25}$, so wird bei Keser über die Embleme des Hipstertums ein mehrheitsgesellschaftlicher Bezug hergestellt.

Die 3. Strategie basiert auf der Beschreibung der Verkörperungen. Charakteristisch für diese Form scheint bei der Darstellung migrantisch markierter Bürgerinnen der Rückgriff auf mehrheitsgesellschaftlich negativ aufgeladene Praktiken $^{26}$ :

\footnotetext{
${ }^{25}$ Der Journalistin speziell dieses Artikels schien es jedoch darum zu gehen, solche semantischen Kurzschlüsse zu konterkarieren, denn das Kopftuch ist hier Teil einer individuellen Lebensgeschichte, eines persönlichen und durchdachten Entschlusses. Damit wird dem weit verbreiteten Antikopftuchdiskurs eine alternative Interpretation entgegengesetzt.

${ }^{26}$ Das wurde auch in anderen von mir durchgeführten Analysen zu Subjektkonstruktionen, z. B. in den Artikeln zum Münchner Bahnhofsviertel und ganz besonders zu den dort anzutreffenden bulgarischen Arbeitsmigrantinnen deutlich. In der Artikelauswahl zu dieser Analyse traf diese Strategie des Weiteren auf die Darstellung des Künstlers Acar oder auch des Jugendclubleiters Dad-
}

Yildirim setzt seinen Körper ein, wenn er spricht, er ist viril, dominant. Er redet laut. Wenn er spricht, erwartet er, dass man ihm zuhört. Und dass kein anderer redet. [... ],,Vor 25000 Zuschauern!“" ruft Yildirim in den Raum. Das ist zwar etwas übertrieben, denn in den Archiven [... ] (SZ, 12. Januar 2016).

Dieses Beispielzitat versammelt eine Auswahl kultureller Stereotype bezüglich sogenannter südländischer Männer. Zentrale Elemente der Charakterisierung sind z. B. die wilde Gestik, die laute Stimme, ein autoritäres männliches Auftreten (Jäger, 2010). Diese Form der Subjektkonstruktion trägt dazu bei, die ansonsten im Text positive Lesart des Protagonisten zu verunsichern, wodurch auch seine Aussagekraft abgeschwächt wird. Dass dies nicht zufällig geschieht, sondern strategisch gesetzt ist, dafür spricht im Artikel über Yildirim, dass er (1) nicht nur einen, sondern gleich mehrere dieser Stereotypen erfüllt, welche (2) in seiner Beschreibung alleinstehend und (3) im gesamten Text verstreut sind. (4) Hebt der Journalist die Beschreibungen immer weiter verallgemeinernd aus dem Moment ihrer gemeinsamen Begegnung heraus, so dass Leserinnen darin auch allgemeine Charakterzüge lesen können. Somit befördern Subjektkonstruktionen dieser Art über die Beschreibung kulturell stereotypisierter Körper und körperlicher Praktiken die ambivalente Wahrnehmung von ansonsten im Artikel positiv dargestellten Akteurinnen.

\section{Resümee}

Meiner diskursanalytischen Sondierung lag die Frage nach den Bedingungen und Möglichkeiten der Partizipation migrantisch markierter Bürgerinnen an den diskursiven Aushandlungen in der Süddeutschen Zeitung zugrunde, der ich beispielhaft an zehn ausgewählten Zeitungsartikeln nachgegangen bin. Im Vordergrund stand dabei die Analyse des Zusammenspiels der zentralen Diskurselemente, die diese Aushandlungen strukturieren: (1) der Gegenstand Migration/Integration, (2) die damit verschränkten Diskursthemen, (3) die dabei verwendeten Begriffe und Klassifikationen und (4) die Charakteristika der Äußerungsmodalitäten. Im letzten Punkt lag ein besonderes Augenmerk auf den Subjektkonstruktionen migrantisch markierter Sprecherinnen. Ziel meiner Analyse war es, die (Macht-)Verhältnisse zwischen Mehrheit und minorisierten Migrantinnen und somit auch die Wirkmächtigkeit spezifischer Wissensbestände, welche die diskursiven Aushandlungen um Migration/Integration in der Süddeutschen Zeitung strukturieren, näher zu beleuchten. Interpretativ leitend war dabei die postmigrantische Debatte, speziell die Konzepte der ,differentiellen Inklusion“ und der

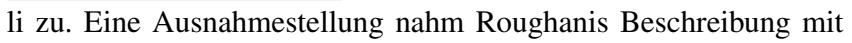
mehrheitsgesellschaftlich positiv konnotierten körperlichen Praktiken ein: „ein groß gewachsener Mann mit dunklen, kurzen Haaren, einem wachen Blick und einem gewinnenden Lächeln“ (SZ, 14. März 2011a). 
„Kämpfe der Migration“ sowie rassismuskritische Fragestellungen.

Meine Analyse hat gezeigt, dass in den diskursiven Aushandlungen zum Gegenstand Migration/Integration der Fokus auf den alltäglichen Folgen des mehrheitsgesellschaftlichen Systems der differentiellen Inklusion für Migrantinnen liegt, das kulturrassistisch strukturiert ist und für die Betroffenen soziale, ökonomische sowie politische Einschränkungen zur Folge hat. Dabei entwirft die Analyse aus der „Perspektive der Migration“ ein Bild von der durch die Gesellschaft gezogenen Segmentierungslinien entlang der Schnittstelle Migrationserfahrung. Die Ergebnisse machen aber auch deutlich, dass migrantisch markierte Bürgerinnen sich in unterschiedlichen Formen im Zeitungsdiskurs dagegen positionieren, u. a. mit eigenen Reflektionen und Interpretationen der Wirklichkeit, mit politischen Forderungen, mit offener Kritik und Witz, Provokationen und eigenen Lebenserfahrungen. Sie nutzen in den ausgewählten zehn Artikeln den mehrheitsgesellschaftlichen Raum der Süddeutschen Zeitung als Angebot für Aushandlungsprozesse mit der Mehrheitsgesellschaft. Sie machen dadurch viele der alltäglichen und für die Mehrheit unsichtbaren Formen und Momente der Minorisierung und der „migrantischen Kämpfe“ für die Mehrheit sichtbar.

Die Möglichkeiten der Partizipation migrantisch markierter Bürgerinnen im mehrheitsgesellschaftlich kontrollierten Feld des Zeitungsdiskurses, die unterschiedlichen journalistischen Rahmungen ihres Sprechens, die Formen ihrer dabei diskursiv evozierten, fragmentarischen Subjektkonstruktionen verweisen auf ihren prekären Sprecherinnenstatus. Denn einerseits brauchen sie diese Unterstützung, um ihrer Perspektive Sagbarkeit zu verleihen und die Normalisierung ihrer Positionen durch den Zeitungsdiskurs zu erreichen. Andererseits kennzeichnet - trotz großer journalistischer Unterstützung und Legitimierung ihrer Sprechakte in meiner Auswahl - nicht selten die widersprüchliche und gegenläufige Verwebung der diskursiven Elemente die journalistischen Texte. Diese diskursive Praxis verleiht den Sprechakten und Positionierungen migrantisch markierter Bürgerinnen wiederum eine Brüchigkeit und Ambivalenz. Dies ist ein zentraler Aspekt der Formation des journalistischen Diskurses in meiner Auswahl. Die spezifischen Figurationen des Sprecherinnenverhältnisses zwischen Mehrheit und migrantisch Minorisierten ermöglicht es, auch in einer als pro-migrantisch zu bezeichnenden Diskursposition - durch die Einführung eines disharmonischen Untertons - einen Kontrapunkt in der Erzählung zu erzeugen, der wiederum hilft, migrationskritische Positionen der Mehrheitsgesellschaft $\mathrm{zu}$ inkludieren und sie nicht zu denormalisieren.

Datenverfügbarkeit. Alle in der Analyse verwendeten Zeitungsartikel sind im Archiv der Süddeutschen Zeitung abrufbar, die jeweilige URL zum Artikel ist in der Literaturliste vermerkt.
Interessenkonflikt. Die Autorin erklärt, dass kein Interessenkonflikt besteht.

Begutachtung. This paper was edited by Benedikt Korf and reviewed by two anonymous referees.

\section{Literatur}

Balibar, E.: The Construction of Racism, Actuel Marx, 2, 11-28, 2005.

Balibar, E.: Europe as Borderland, Environ. Plan. D, 27, 190-215, 2009.

Bauriedl, S.: Räume lesen lernen: Methodeneinsatz für eine Diskursanalyse räumlicher Differenzierung, Forum Qualitative Sozialforschung, 8, 13, 2007.

Binder, B.: Beheimatung statt Heimat: Translokale Perspektiven auf Räume der Zugehörigkeit, in: Zwischen Emotion und Kalkül. ,Heimat‘ als Argument im Prozess der Moderne, Herausgeber: Seifert, M., Leipziger Universitätsverlag, Germany, 189204, 2010.

Blum, R.: Einleitung. Leidende Leuchttürme. Über die Unentbehrlichkeit von Qualitätsmedien, in: Krise der Leuchttürme öffentlicher Kommunikation. Vergangenheit und Zukunft der Qualitätsmedien, Herausgeber: Blum, R., Bonfadelli, H., Imhof, K. und Jarren, O., Springer VS, Heidelberg, Germany, 7-14, 2011.

Bojadžijev, M. und Römhild, R.: Was kommt nach dem ,transnational turn"? Perspektiven für eine kritische Migrationsforschung, in: Vom Rand ins Zentrum. Perspektiven einer kritischen Migrationsforschung, Herausgeber: Labor Migration, Panama Verlag, Berlin, Germany, 10-24, 2014.

Broden, A. und Mecheril, P.: Migrationsgesellschaftliche RePräsentationen. Eine Einführung, in: Re-Präsentationen. Dynamiken der Migrationsgesellschaft, Herausgeber: Broden, A. und Mecheril, P., IDA-NRW, Düsseldorf, Germany, 7-28, 2007.

Broden, A. und Mecheril, P.: Rassismus bildet. Einleitende Bemerkungen, in: Rassismus bildet, in: Bildungswissenschaftliche Beiträge zu Normalisierung und Subjektivierung in der Migrationsgesellschaft, Herausgeber: Broden, A. und Mecheril, P., transcript, Bielefeld, Germany, 7-23, 2010.

Butler, J.: Haß spricht - Zur Politik des Performativen, Suhrkamp, Frankfurt am Main, Germany, 2006.

Canan, C. und Foroutan, N.: Deutschland postmigrantisch III. Migrantische Perspektiven auf deutsche Identitäten - Einstellungen von Personen mit und ohne Migrationshintergrund zu nationaler Identität in Deutschland, Berlin, Germany, online aufrufbar: https://www.projekte.hu-berlin.de/de/junited/ deutschland-postmigrantisch-3.pdf (letzter Zugriff: 23. Juni 2020), 2016.

De Certeau, M.: Kunst des Handelns, Merve, Berlin, Germany, 1988.

Diaz-Bone, R.: Kulturwelt, Diskurs und Lebensstil. Eine diskurstheoretische Erweiterung der bourdieuschen Distinktionstheorie, Leske + Budrich, Opladen, Germany, 2002.

Diaz-Bone, R.: Zur Methodologisierung der Foucaultschen Diskursanalyse, Hist. Social Res., 31, 243-274, https://doi.org/10.12759/hsr.31.2006.2.243-274, 2006. 
El-Tayab, F.: European Others: Queering Ethnicity in Postnational Europe, University of Minnesota Press, Minneapolis, USA, 2011.

Espahangizi, K., Hess, S., Karakayali, J., Kasparek, B., Pagano, S., Rodatz, M., und Tsianos, V. S.: Rassismus in der postmigrantischen Gesellschaft. Zur Einleitung, movements. Journal für kritische Migrations- und Grenzregimeforschung, 2, 1, transcript, Bielefeld, Germany, 9-23, 2016.

Foroutan, N.: Was will eine postmigrantische Gesellschaftsanalyse?, in: Postmigrantische Perspektiven. Ordnungssysteme, Repräsentationen, Kritik, Herausgeber: Foroutan, N., Karakayali, J., und Spielhaus, R., Campus, Frankfurt, Germany, 269-299, 2018.

Foucault, M.: Die Ordnung des Diskurses, Fischer TBV, Frankfurt, Germany, 7-49, 1994.

Geulen, C.: Geschichte des Rassismus, C. H. Beck, München, Germany, 2007.

Honneth, A.: Organisierte Selbstverwirklichung. Paradoxien der Individualisierung, in: Befreiung aus der Mündigkeit. Paradoxien des gegenwärtigen Kapitalismus, Herausgeber: Honneth, A., Campus, Frankfurt am Main, Germany, 141-158, 2002.

Jäger, M.: Rassismus und Normalität im Alltagsdiskurs. Anmerkungen zu einem paradoxen Verhältnis, in: Rassismus bildet, in: Bildungswissenschaftliche Beiträge zu Normalisierung und Subjektivierung in der Migrationsgesellschaft, Herausgeber: Broden, A. und Mecheril, P., transcript, Bielefeld, Germany, 27-39, 2010.

Jäger, M. und Jäger, S.: Deutungskämpfe. Theorie und Praxis kritischer Diskursanalyse, VS Verlag, Wiesbaden, Germany, 2007.

Jäger, S.: Kritische Diskursanalyse: Eine Einführung, Unrast Verlag, Münster, Germany, 2015.

Lanz, S.: Berlin aufgemischt: abendländisch, multikulturell, kosmopolitisch? Die politische Konstruktion einer Einwanderungsstadt, transcript, Bielefeld, Germany, 2007.

Lanz, S.: In unternehmerische Subjekte investieren. Integrationskonzepte im Workfare-Staat. Das Beispiel Berlin, in: No integration?! Kulturwissenschaftliche Beiträge zur Integrationsdebatte in Europa, Herausgeber: Hess, S., Binder, J., und Moser, J., transkript, Bielefeld, Germany, 105-122, 2009.

Marche, G.: Why infrapolitics matters, revue francaise detudes americaines, online aufrufbar: https://www.cairn.info/ revue-francaise-d-etudes-americaines-2012-1-page-3.htm (letzter Zugriff: 23. Juni 2020), 2012.

Mezzadra, S. and Neilson, B.: Border as method, or, the multiplication of labor, Duke University Press, Durham, UK, 2013.

Münch, S.: Integration durch Wohnungspolitik? Zum Umgang mit ethnischer Segregation im europäischen Vergleich, VS Verlag, Wiesbaden, Germany, 2010.

Pütz, R. und Rodatz, M.: Kommunale Integrations- und Vielfaltskonzepte im Neoliberalismus. Zur strategischen Steuerung von Integration in deutschen Großstädten, Geogr. Z., 101, 166-183, 2013.

Riedner, L.: Arbeit! Wohnen! Urbane Auseinandersetzungen um EU-Migration. Eine Untersuchung zwischen Wissenschaft und Aktivismus, edition assemblage, Münster, Germany, 2018.

Rodatz, M.: Migration ist in dieser Stadt eine Tatsache. Urban politics of citizenship in der neoliberalen Stadt, suburban zeitschrift für kritische stadtforschung, 2, 35-58, https://doi.org/10.36900/suburban.v2i3.155, 2014.
Römhild, R.: Aus der Perspektive der Migration: Die Kosmopolitianisierung Europas, Argument, 285, 50-59, 2010.

Römhild, R.: Beyond the bounds of the ethnic: for postmigrant cultural and social research, J. Aesthet. Cult., 9, 2, 69-75, https://doi.org/10.1080/20004214.2017.1379850, 2017.

Ronneberger, K. und Tsianos, V.: Panische Räume. Das Ghetto und die „Parallelgesellschaft,,, in: No integration?! Kulturwissenschaftliche Beiträge zur Integrationsdebatte in Europa, Herausgeber: Hess, S., Binder, J. und Moser, J., transkript, Bielefeld, Germany, 137-152, 2009.

Rosol, M.: Regieren in der neoliberalen Stadt. Foucaults Analyse des Neoliberalismus als Beitrag zur Stadtforschung, Geogr. Z., 101, 132-147, 2013.

Scheel, S.: Das Konzept der Autonomie der Migration überdenken? Yes, please!, movements, Journal für kritische Migrations- und Grenzregimeforschung, online aufrufbar: https://movements-journal.org/issues/02.kaempfe/14. scheel--autonomie-der-migration.html (letzter Zugriff: 23. Juni 2020), 2015.

Schrödter, M.: Die Objektivität des Rassismus. Anerkennungsverhältnisse und prekäre Identitätszumutungen, in: Re-Präsentationen. Dynamiken der Migrationsgesellschaft, Herausgeber: Broden, A. und Mecheril, P., IDA-NRW, Düsseldorf, Germany, 69-93, 2007.

Spielhaus, R.: Studien in der postmigrantischen Gesellschaft. Eine kritische Auseinandersetzung, in: Kongressdokumentation 4 . Bundesfachkongress Interkultur_DIVERCITY_Hamburg_24.-26.10.2012,

Kulturbehörde der Freien und Hansestadt Hamburg, Hamburg, Germany, 96-100, online aufrufbar: https: //www.bundesfachkongress-interkultur-2012.de/ (letzter Zugriff: 23. Juni 2020), 2012.

Spielhaus, R.: Zwischen Migrantisierung von Muslimen und Islamisierung von Migranten, in: Postmigrantische Perspektiven. Ordnungssysteme, Repräsentationen, Kritik, Herausgeber: Foroutan, N., Karakayali, J., und Spielhaus, R., Campus, Frankfurt, Germany, 129-143, 2018.

SZ - Süddeutsche Zeitung: Schreibwerkstatt Migrantinnen. Geschichten aus der neuen Heimat, online aufrufbar: https://www.sueddeutsche.de/muenchen/landkreismuenchen/ schreibwerkstatt-migrantinnen-schreibwerkstatt-migrantinnen-1. 978369 (letzter Zugriff: 28. Juni 2018), 17. August 2010.

SZ - Süddeutsche Zeitung: Migranten in München (3) Die Stimme der Minderheit, online aufrufbar: https://www.sueddeutsche.de/muenchen/ migranten-in-muenchen-die-stimme-der-minderheit-1.1028249 (letzter Zugriff: 28. Juni 2018), 10. Dezember 2010.

SZ - Süddeutsche Zeitung: Migranten in München (1) Tuncay Acar „Klingt wie Zylinderkopfdichtung“, online aufrufbar: https://www.sueddeutsche.de/muenchen/landkreismuenchen/migranten (letzter Zugriff: 28. Juni 2018), 14. März 2011a.

SZ - Süddeutsche Zeitung: Migranten in München (5) In Zahlen Stadt der Vorzeigemigranten, online abrufbar: https://www.sueddeutsche.de/muenchen/landkreismuenchen/ migranten-in-muenchen-5-in-zahlen-stadt-der-vorzeigemigranten-1. 1033640 (letzter Zugriff: 23. Juni 2020), 14. März 2011b.

SZ - Süddeutsche Zeitung: Migranten in München (8) Amir Roughani Typisch deutsch!, online aufrufbar: https://www.sueddeutsche.de/muenchen/landkreismuenchen/ 
migranten-in-muenchen-amir-roughani-typisch-deutsch-1. 1058472 (letzter Zugriff: 28. Juni 2018), 14. März 2011c.

SZ - Süddeutsche Zeitung: Oberschleißheim. als ein Jugendzentrum, online aufrufbar: https: //www.sueddeutsche.de/muenchen/landkreismuenchen/ oberschleissheim-mehr-als-ein-jugendzentrum-1.2719204 (letzter Zugriff: 28. Juni 2018), 2. November 2015.

SZ - Süddeutsche Zeitung: Preis für MigrantenUnternehmen. Auberginen als Medizin, online aufrufbar: https://www.sueddeutsche.de/muenchen/ preis-fuer-migranten-unternehmen-auberginen-als-medizin- 1 . 2815181 (letzter Zugriff: 28. Juni 2018), 12. Januar 2016.

SZ - Süddeutsche Zeitung: Vor 15 Jahren kam er zum Studium. „Ich fühle mich hier beheimatet“, online aufrufbar: https://www.sueddeutsche.de/muenchen/ vor-jahren-kam-er-zum-studium-ich-fuehle-mich-hier-beheimatet- 1. 2877215 (letzter Zugriff: 28. Juni 2018), 23. Februar 2016.

SZ - Süddeutsche Zeitung: Erfahrungen mit Ausgrenzung. „Das ist doch mein Zuhause!“, online aufrufbar: https://www.sueddeutsche.de/muenchen/ erfahrungen-mit-ausgrenzung-das-ist-doch-mein-zuhause- 1 . 3063013 (letzter Zugriff: 28. Juni 2018), 4. Juli 2016.
SZ - Süddeutsche Zeitung: Unterschleißheim. Ratloser Beirat, online aufrufbar: https://www.sueddeutsche.de/muenchen/ landkreis-muenchen/unterschleissheim-ratloser-beirat- 1 . 3123751 (letzter Zugriff: 28. Juni 2018), 17. August 2016.

SZ - Süddeutsche Zeitung: Neuperlach. „Ich spüre die abschätzigen Blicke“, online aufrufbar: https://www.sueddeutsche.de/ muenchen/neuperlach-ich-spuere-die-abschaetzigen-blicke- 1. 3261275 (letzter Zugriff: 28. Juni 2018), 22. November 2016.

Tsianos, V.: Urbane Paniken. Zur Entstehung des antimuslimischen Urbanismus, in: Wer Macht Demokratie? Kritische Beiträge zu Migration und Machtverhältnissen, Herausgeber: Gürsel, D., Zülfukar, Ç., und Allmende, E. V., edition assemblage, Münster, Germany, 23-43, 2013.

Velho, A.: (Un-)Tiefen der Macht. Subjektivierung unter den Bedingungen von Rassismuserfahrungen in der Migrationsgesellschaft, in: Rassismus bildet. Bildungswissenschaftliche Beiträge zu Normalisierung und Subjektivierung in der Migrationsgesellschaft, Herausgeber: Broden, A. und Mecheril, P., transcript, Bielefeld, Germany, 113-137, 2010.

Wacquant, L.: Das Janusgesicht des Ghettos und andere Essays, Birkhäuser, Basel, Schweiz, 2006. 